\title{
Multiwall Carbon Nanotube Modified Electrochemical Sensor for Reactive Black 5
}

\author{
Velliangiri Sreeja ${ }^{1}$, Raman Sasikumar ${ }^{2}$, Marimuthu Alagarsamy ${ }^{2}$ Paramasivam Manisankar $^{2 *}$ \\ ${ }^{1}$ Vellalar College for Women (Autonomous), Tamil Nadu, India \\ ${ }^{2}$ Department of Industrial Chemistry, Alagappa University, Tamil Nadu, India \\ E-mail: ${ }^{*}$ pms11@rediffmail.com \\ Received July 18, 2011; revised August 19, 2011; accepted August 29, 2011
}

\begin{abstract}
Cyclic voltammograms of reactive black5 (RB5) at different $\mathrm{pHs}$ in the range 1.0 - 13.0 on multiwall carbon nanotube modified glassy carbon electrode revealed the presence of one well-defined irreversible anodic peak around $975 \mathrm{mV}$ in acidic and neutral pHs. Adsorption controlled oxidation observed at acidic $\mathrm{pH} 1.0$ resulted in the maximum peak current response in cyclic voltammograms. A systematic differential pulse stripping voltammetric studies were carried out using the modified electrode at $\mathrm{pH}$ 1.0. The accumulation parameters, accumulation potential and time were optimized for maximum adsorption of the dye which was ascertained from the SEM photographs and XRD results. The stripping parameters were optimized and calibration was made under optimum conditions. The range of study was from $0.5 \mathrm{ppm}$ to $100 \mathrm{ppm}$ and the lower limit of determination was $100 \mathrm{ppm}$. Five identical experiments were carried out and the RSD value obtained was $2.5 \%$ suggesting good reproducibility. The proposed method was successfully applied to determine the concentration of dye in the fabric and wastewater after dyeing.
\end{abstract}

Keywords: Cyclic Voltammetry, Reactive Black 5, Stripping Voltammetry, Multiwall Carbon Nanotubes

\section{Introduction}

An important milestone in the history of carbon materials is the discovery of carbon nanotubes (CNTs) [1] having two distinct types of structures namely single walled and multiwalled. As a consequence of the excellent electronic and conducting properties of CNTs, electrodes modified with CNTs have demonstrated to improve the electroanalytical performance of different species. Due to their uniqueness, CNTs have received enormous attention for the preparation of electrochemical sensors as it was extensively reviewed [2-5]. The subtle electronic behavior of CNTs reveals that they have the ability to promote electron-transfer reaction when used as electrode materials. Recently CNT film coated electrodes have received increasing attention in analytical studies [7-9]. However a major barrier for developing the CNT modified electrode is the insolubility of CNTs in usual media [10] and many efforts have been made to disperse CNTs into suitable solvents such as DMF [11], acetone [12] and concentrated sulphuric acid [13]. Yuan-hai Zhu et al. [14] functionalized MWCNTs using nitrating mixture and neutralized with dil. $\mathrm{NaOH}$. The modified MW-
CNTs were water soluble and used for the determination of phenylephrine. In recent days, a noncovalent method [15] has been developed and ported for solubilizing MWCNTs functionalized with Congo red. Surfactants are a special kind of amphiphilic molecules, which can spontaneously adsorb at the interfaces or assemble into micelles in solutions, forming various regulated structures at electrode surfaces or in solutions. This resulted in extensive applications in electroanalysis [16]. MWCNTs modified electrodes fabricated in the presence of surfactants resulted in high sensitivity and selectivity. MWCNT/GCE modified electrode fabricated in the pre sence of SDS exhibited enhanced sensing of organic pollutants $[17,18]$. Hence the present work, we used anionic surfactant, sodium dodecyl sulphate (SDS) to disperse MWCNTs.

Reactive dyes are the main group of dyes used in the textile industry [19]. They are very effective in fabric dyeing due to the reactive groups capable of forming covalent bonds with a hydroxyl or amino group on the fiber. Inefficiency in the dyeing process resulted in $10 \%-15 \%$ of all dyestuff being lost directly to wastewater [20]. Billions of kilograms of dyes are produced per annum 
and are used in diverse applications including textile dyes, paints, pigments, printing inks and food colouring. In general about $20 \%$ of dye loses would have entered the environment via the wastewater treatment facilities $[21,22]$. In that water body dyes have been shown potentially to have a long half-life in the environment. Analytical chemistry in pollution control is playing an everincreasing vital role in international trade and industry. The confidence and reliability of analytical results must play a major part in world trade and industrial pollution control. The methods used for monitoring these dyes during dyeing and washing are generally based on chromatography and spectrophotometry [23]. The determination of dyes by spectrophotometry has presented special problems owing to lack of selectivity and sensitivity. Additional complications are noticed in chromatography as this group of compounds is usually ionic and of high polarity, as well as nonvolatile and thermally unstable. HPLC, at its current stage of development, is clearly not a method for analytical problems with a high repetition rate because the receptive condition of the system requires 24 to 36 hours. On the other hand, electroanalysis is a manageable method, which is suitable for various problems [24]. Reactive Black5 (RB5) is a commonly and widely used reactive dye and hence development of sensitive stripping voltammetric method for its determination using MWCNT modified GCE is undertaken.

\section{Experimental}

\subsection{Reagents and Apparatus}

Multi-Walled CNTs (I.D.x length $(2-15) \mathrm{nm} \times(1-10)$ $\mu \mathrm{m}$, produced by arc method) purchased from the Sigma Aldrich and AR sodium dodecyl sulphate (SDS) from Merck. The reactive black 5 was obtained from Astick Dyestuff pvt. Ltd, Mumbai, India. The stock solution was prepared by dissolving the substance in double distilled water purified from TKA purification system. For studies aqueous media, $0.1 \mathrm{M} \mathrm{H}_{2} \mathrm{SO}_{4}$ (for $\mathrm{pH} 1.0$ ), Britton Robinson Buffers (for $\mathrm{pH} 4.0,7.0,9.2$ ) and $0.1 \mathrm{M}$ $\mathrm{NaOH}$ (for $\mathrm{pH} 13.0$ ) were used. The electrochemical studies were performed with a CHI $760 \mathrm{C}$ electrochemical workstation (CH Instruments, USA). The MWCNT /GCE was working electrode. Platinum wire and $\mathrm{Ag} /$ $\mathrm{AgCl}$ were employed as an auxiliary and reference electrode respectively. To get reproducible results, great care was taken in the electrode pretreatment.

\subsection{Fabrication of MWCNT/GCE}

$1 \mathrm{mg}$ MWCNT was dispersed in $1 \mathrm{ml}$ of $0.1 \mathrm{M}$ sodium dodecyl sulphate using an ultrasonicator to give black suspensions. Cast films were prepared by placing $5 \mu \mathrm{L}$ of the MWCNT/surfactant suspension on GCE and then evaporating it in an oven at $50^{\circ} \mathrm{C}$.

\section{Results and Discussion}

\subsection{Cyclic Voltammetric Studies of RB5}

Cyclic voltammogram of RB5 using GCE and MWCNT/ GCE were recorded and presented Figure 1. Only one oxidation peak was observed in both cyclic voltammograms. The oxidation peak appeared at lower potential with higher current when MWCNT/GCE was employed. This indicates facile oxidation at this modified electrode and hence develop voltammetric studies of RB5 passed using MWCNT/GCE. The oxidation peak was found to shift anodically with increase in scan rate. The plot of peak current versus scan rate resulted in straight line with good correlation whereas lesser correlation was observed between Peak current and square root of scan rate. Suggesting adsorption-controlled oxidation. Log of peak currents were correlated with the log of scan rate and it resulted in straight line with slope 0.6004, conforming adsorption-controlled oxidation of RB5 in this acidic $\mathrm{pH}$. Absence of peak in the reverse scan and fractional $\alpha$ n value determined from slope of the plot Ep vs. $\log v$ revealed irreversible oxidation. The dye concentration was varied from $300 \mathrm{ppm}$ to $1000 \mathrm{ppm}$ at constant scan rate of $100 \mathrm{mV} / \mathrm{s}$. The peak potential of the anodic peak increased with increase in concentration. The peak current also showed increasing trend with increase in concentration. Adsorption and higher current response in the $\mathrm{cv}$ studies suggested the development of adsorptive stripping procedure for the determination of RB5.

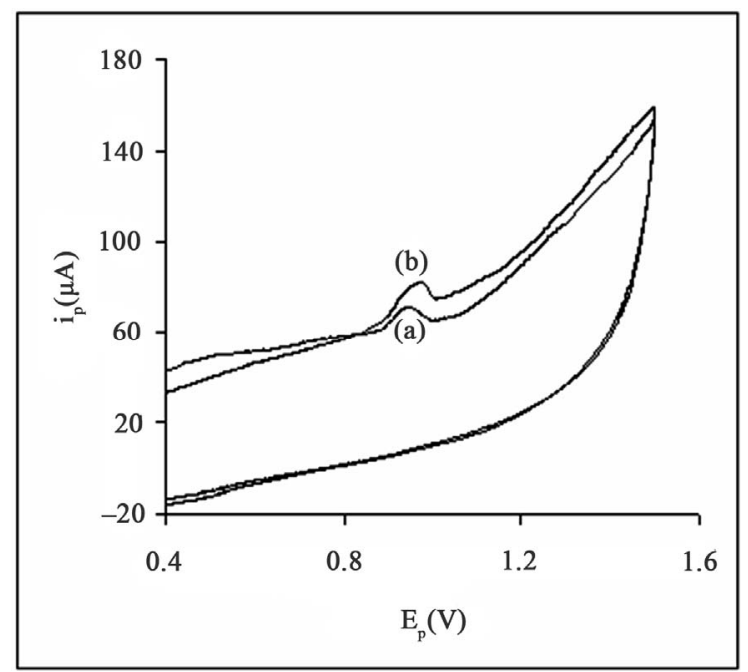

Figure 1. Cyclic voltammogram of 500 ppm RB5 on (a) GCE; (b) MWCNTs/GCE at $\mathrm{pH}-1.0$ scan rate $100 \mathrm{mV} / \mathrm{s}$. 


\subsection{Adsorption of RB5 on MWCNT/GCE}

SEM and XRD analysis. The SEM micrographs were recorded for the MWCNT/GCE surface and adsorbed RB5 and presented in Figures 2(a)-(b) respectively. The SEM figure of MWCNT/GCE is entirely different from that of dye adsorbed. Figure 2(b) shows well developed rod like structure for the dye with approximately $100 \mathrm{~nm}$ dia. Comparison of the two SEMs confirms the strong adsorption of the dye on MWCNT/GCE. The X-ray diffractograms of the MWCNT/GCE and RB5 adsorbed on MWCNT/GCE are presented in Figures 3(a)-(b) respectively. The XRD results reveal different semi-cry- stalline nature of both.

\subsection{Differential Pulse Stripping Voltammetry}

The Differential pulse stripping voltammetric behaviour of RB5 is shown in Figure 4. At this accumulation po- tential, accumulation time was varied between $5 \mathrm{~s}$ and 40 $\mathrm{s}$ and maximum peak current in the DPSV was observed at $30 \mathrm{~s}$ under the optimum accumulation conditions, RB5 was accumulated and the adsorption of RB5 was confirmed by many preconcentration and stripping voltammetric were investigations were performed for accumulation potentials $\left(\mathrm{E}_{\mathrm{acc}}\right)$ varying from $-1000 \mathrm{mV}$ to 1000 $\mathrm{mV}$ at an accumulation time of $15 \mathrm{~s}$. Differential pulse stripping voltammograms (DPSV) were recorded and maximum Peak current was observed. Which was fixed as the optimum accumulation potential. Since the dye molecule is anionic due to the presence of sulphonate ion in the dye RB5, the effective adsorptive accumulation is in the positive potential. Differential pulse stripping voltammograms were recorded for the adsorbed RB5 under optimized accumulation conditions by varying pulse amplitude maximum peak current conditions were found out and the results are presented in Table 1.
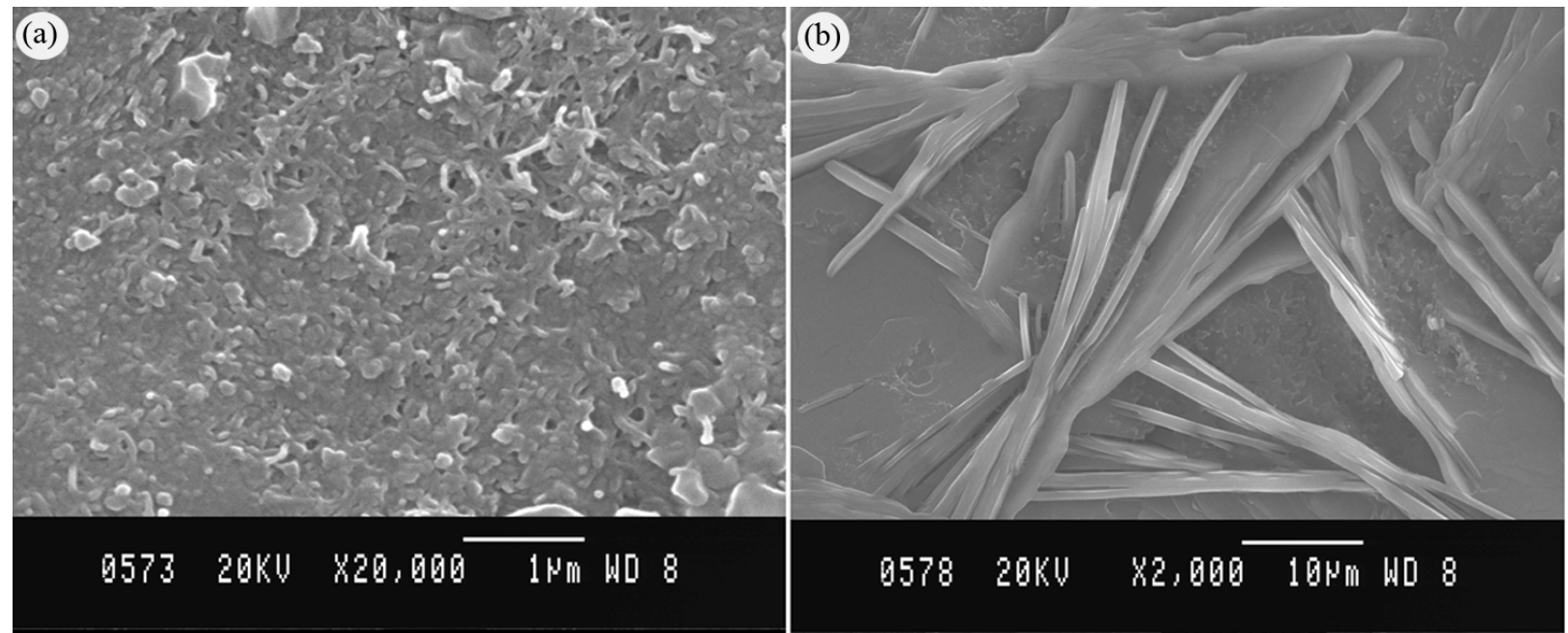

Figure 2. SEM photographs of (a) MWCNTs deposited on GCE; (b) RB5 deposited on MWCNTs/GCE.

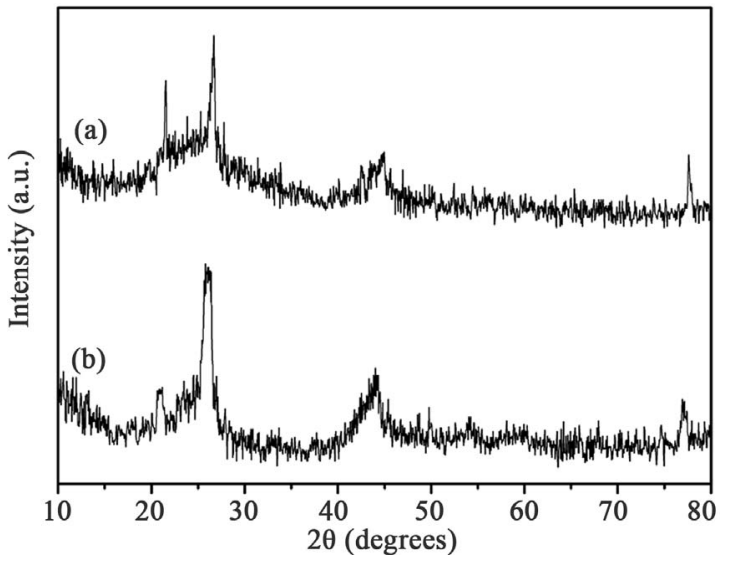

Figure 3. XRD behaviour of (a) MWCNTs deposited on GCE; (b) RB5 deposited on MWCNTs deposited on GCE.

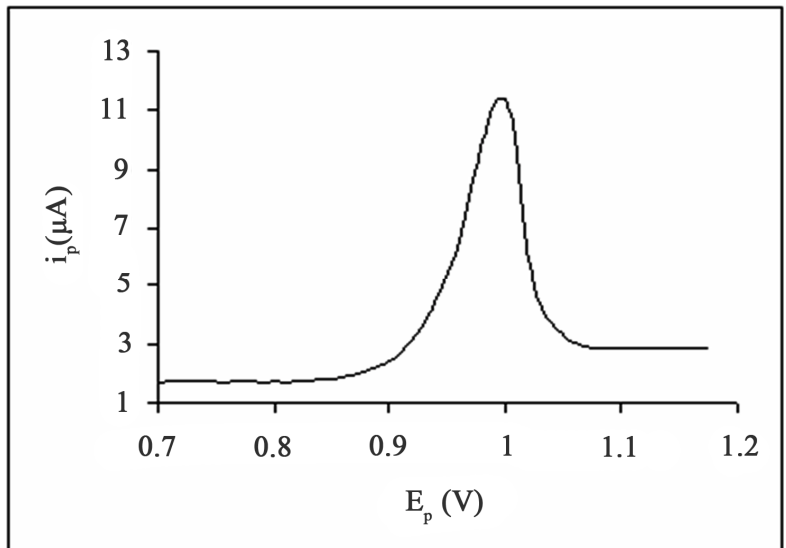

Figure 4. DPSV of $0.3 \mathrm{ppm}$ RB5 standard sample at pH 1.0 under optimum condition. 
Table 1. Optimum experimental conditions of RB5 for differential pulse stripping voltammetry.

\begin{tabular}{ccc}
\hline Variables & Range examined & Optimum value \\
\hline Deposition potential $(\mathrm{mV})$ & -1000 to 1000 & 0 \\
Deposition time $(\mathrm{Sec})$ & 5 to 40 & 30 \\
Amplitude $(\mathrm{mV})$ & 25 to 150 & 100 \\
Pulse width $(\mathrm{mSec})$ & 25 to 150 & 100 \\
Scan increment $(\mathrm{mV})$ & 4 to 10 & 10 \\
\hline
\end{tabular}

\subsection{Analytical Characteristics}

Differential pulse stripping voltammograms at different concentrations of RB5 were recorded under maximum peak current conditions. From the results, a linear calibration graph was obtained (Figure 5) indicating linear dependence between the two. The range of determination was found between $0.5 \mathrm{ppm}$ to $100 \mathrm{ppm}$. The limit of detection was $100 \mathrm{ppb}$. The reproducibility of stripping signal was realized in terms of relative standard deviation for 5 identical measurements carried out and found to be $2.5 \%$.

\subsection{Determination of RB5 in the Wastewater}

To validate the proposed method for the determination of RB5 on real samples, the dye content in the wastewater obtained from the lab scale dying process was determined by employing the calibration plot. A cotton fabric was dyed with RB5 in the laboratory as per the procedure described here. For the dyeing process, the dye bath was set with $0.5 \mathrm{~g}$ of the fabric at $40^{\circ} \mathrm{C}$ for 15 minutes. Additions of $0.5 \mathrm{ml}$ of $5 \%$ sodium carbonate solution, 1 $\mathrm{ml}$ of $5 \%$ dye solution, $0.5 \mathrm{ml}$ of $3 \%$ sodium chloride solution, $0.5 \mathrm{ml}$ of wetting agent were added. The material to liquor (MLR) ratio was kept at 1:25 and the total volume was kept at $50 \mathrm{ml}$. After the dyeing, the dyed cotton fabric was taken out, cooled, washed with cold water and dried. The spent dye liquor or wastewater of the dye bath after dying was collected from the laboratory scale dying unit.

The spent dye liquor was subjected to stripping analysis under optimized conditions proposed from the DPSV studies. The spent dye liquor containing the unspent dye was made acidic by adding $0.1 \mathrm{M} \mathrm{H}_{2} \mathrm{SO}_{4}$ and the total volume was kept at $50 \mathrm{ml}$. The $\mathrm{pH}$ of the solution was ascertained and kept at 1.0.10 $\mathrm{ml}$ of this solution was taken in the cell and the DPSV experiment was carried out under optimum conditions using MWCNT modified glassy carbon electrode. The differential pulse stripping voltammogram is presented in Figure 6. The stripping peak current was measured and substituted in the calibration equation. Thus the amount of RB5 present in the spent dye liquor was determined. The same DPSV determination was repeated for 6 times and the amount of RB5 was determined in each experiment. With the RSD value of $2.9 \%$, the concentration of the dye in the spent dye liquor was determined to be $9.2 \pm 0.2 \mathrm{ppm}$ with the help of the calibration plot.

\section{Conclusions}

Based on this study, it is concluded that the adsorptive stripping voltammetric measurements of RB5 on MWCNT/GCE has resulted in an efficient method for the determination of RB5. The range of determination was found in between $0.5 \mathrm{ppm}$ to $100 \mathrm{ppm}$. The limit of detection was $100 \mathrm{ppb}$. High sensitivity, good reproducibility

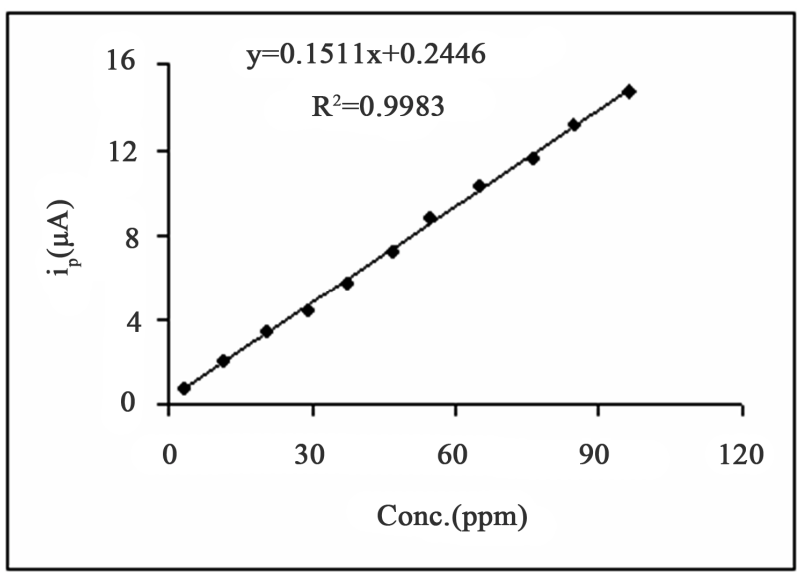

Figure 5. Calibration plot of DPSV of RB5 at pH 1.0 under optimum condition.

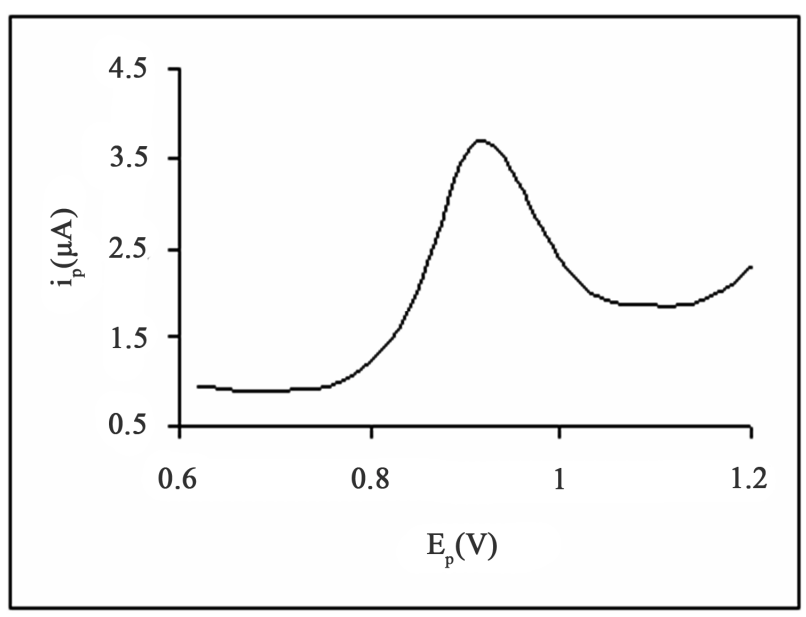

Figure 6. DPSV of $0.3 \mathrm{ppm}$ RB5 real sample at pH 1.0 under optimum condition. 
and simple instrumentation are the added advantages. This method can be easily applied for the determination dye in the waste water.

\section{Acknowledgements}

Mr. R. Sasikumar acknowledges UGC, New Delhi for providing Research Fellowship in Science for Meritorious Students.

\section{References}

[1] S. Iijima, "Helical Microtubules of Graphitic Carbon," Nature, Vol. 354, No. 6348, 1991, pp. 56-58. doi: $10.1038 / 354056 \mathrm{a} 0$

[2] J. Wang, "Carbon-Nanotube Based Electrochemical Biosensors," Electroanalysis, Vol. 17, No. 1, 2005, pp. 7-14. doi:10.1002/elan.200403113

[3] C. E. Banks and R. G. Compton, "New Electrode for Old from Carbon Nanotubes to Edge Plan Phyrolytic Graphite," Analyst, Vol. 131, No. 1, 2006, pp. 15-21. doi:10.1039/b512688f

[4] J. Wang, "Nanomaterial-Based Electrochemical Biosensors," Analyst, Vol. 130, No. 4, 2005, pp. 421-426. doi: $10.1039 / \mathrm{b} 414248 \mathrm{a}$

[5] M. Valcarcel, B. M. Simonet, S. Cardenas and E. B. Suarez, "Present and Future Applications of Carbon Nanotubes to Analytical Science," Analytical and Bioanalytical Chemistry, Vol. 382, No. 8, 2005, pp. 17831790. doi:10.1007/s00216-005-3373-3

[6] Q. Honglan and Z. Chengxiao, "Simultaneous Determination of Hydroquinone and Catechol at a Glassy Carbon Electrode Modified with Multiwall Carbon Nanotubes," Electroanalysis, Vol. 17, No. 10, 2005, pp. 832-838. doi:10.1002/elan.200403150

[7] H. Zhang, C. Hu, S. Wu and S. Hu, "Enhanced Oxidation of Simvastatin at a Multi-Walled Carbon NanotubesDihexadecyl Hydrogen Phosphate Composite Modified Glassy Carbon Electrode and the Application in Determining Simvastatin in Pharmaceutical Dosage Forms," Electroanalysis, Vol. 17, No. 9, 2005, pp. 749-754. doi:10.1002/elan.200403137

[8] S. L. Nathan, P. D. Randhir and J. Wang, "Comparison of the Electrochemical Reactivity of Electrodes Modified with Carbon Nanotubes from Different Sources," Electroanalysis, Vol. 17, No. 1, 2005, pp. 65-72. doi:10.1002/elan.200403120

[9] K. A. Joshi, J. Tang, R. Haddon, J. Wang, W. Chen and A. Mulchandani, "A Disposable Biosensor for Organophosphorus Nerve Agents Based on Carbon Nanotubes Modified Thick Film Strip Electrode," Electroanalysis, Vol. 17, No. 1, 2005, pp. 54-58. doi:10.1002/elan.200403118

[10] J. Wang, M. Musameh and Y. Lin, "Solubilization of Carbon Nanotubes by Nafion toward the Preparation of Am- perometric Biosensors," Journal of the American Chemical Society, Vol. 125, No. 9, 2003, pp. 2408-2409. doi:10.1021/ja028951v

[11] H. X. Luo, Z. J. Shi, N. Q. Li, Z. N. Gu and Q. K. Zhuang, "Investigation of the Electrochemical and Electrocatalytic Behavior of Single-Wall Carbon Nanotube Film on a Glassy Carbon Electrode," Analytical Chemistry, Vol. 73, No. 5, 2001, pp. 915-920. doi:10.1021/ac0009671

[12] F. H. Wu, G. C. Zhao and X. W. Wei, "Electrocatalytic Oxidation of Nitric Oxide at Multi-Walled Carbon Nanotubes Modified Electrode," Electrochemistry Communications, Vol. 4, No. 9, 2002, pp. 690-694. doi:10.1016/S1388-2481(02)00435-6

[13] M. Musamech, J. Wang, A. Merkoci and Y. H. Lin, "LowPotential Stable NADH Detection at Carbon-NanotubeModified Glassy Carbon Electrodes," Electrochemistry Communications, Vol. 4, No. 10, 2002, pp. 743-752. doi:10.1016/S1388-2481(02)00451-4

[14] Y. Zhu, Z. Zhang, W. Zhao and D. Pang, "Voltammetric Behavior and Determination of Phenylephrine at a Glassy Carbon Electrode Modified with Multi-Wall Carbon Nanotubes," Sensors and Actuators B, Vol. 119, No. 1, 2006, pp. 308-314. doi:10.1016/j.snb.2005.12.026

[15] C. Hu, C. Yang and S. Hu, "Hydrophobic Adsorption of Surfactants on Water-Soluble Carbon Nanotubes: A Simple Approach to Improve Sensitivity and Antifouling Capacity of Carbon Nanotubes-Based Electrochemical Sensors," Electrochemistry Communications, Vol. 9, No. 1, 2007, pp. 128-134. doi:10.1016/j.elecom.2006.08.055

[16] R. Vittal, H. Gomathi and K. J. Kim, "Beneficial Role of Surfactants in Electrochemistry and in the Modification of Electrodes," Advances in Colloid and Interface Science, Vol. 119, No. 1, 2006, pp. 55-58. doi:10.1016/j.cis.2005.09.004

[17] P. Manisankar, P. L. A. Sundari, R. Sasikumar and S. P. Palaniappan, "Electroanalysis of Some Common Pesticides Using Conducting Polymer/Multiwalled Carbon Nanotubes Modified Glassy Carbon Electrode," Talanta, Vol. 76, No. 5, 2008, pp. 1022-1028. doi:10.1016/j.talanta.2008.04.056

[18] P. Manisankar, P. A. Sundari and R. Sasikumar, "SquareWave Stripping Voltam-Metric Determination of Some Organic Pollutants Using Modified Electrodes," International Journal of Environmental Analytical Chemistry, Vol. 89, No. 4, 2009, pp. 245-260. doi: $10.1080 / 03067310802658440$

[19] H. Zollinger, "Color Chemistry," 2nd Edition, V. C. H. Publisher, New York, 1991.

[20] K. Venkataraman, "The Chemistry of Synthetic Dyes," Academic Press, New York, 1972.

[21] E. A. Clarke and R. Anliker, "Organic Dyes and Pigments," In: O. Hutzinger Ed., Handbook of Environmental Chemistry, Vol. 3, Part A, Springer-Verlag, Berlin, 1980, pp. 181-215.

[22] E. J. Weber and V. C. Stickney, "Hydrolysis Kinetics of Reactive Blue 19-Vinyl Sulfone," Water Research, Vol. 
27, No. 1, 1993, pp. 63-67. doi:10.1016/0043-1354(93)90195-N

[23] C. Chritodoultos and D. A.Vaccari, "Correlations of Performance for Activated Sludge Using Multiple Regression with Autocorrelation," Water Research, Vol. 27, No. 1, 1993, pp. 51-62. doi:10.1016/0043-1354(93)90194-M

[24] R. Kalvoda and R. Parsons, "Electrochemistry in Research and Development," Plenum Press, New York, 1985. 